\title{
The Whole Zone Apparent Resistivity Calculation Method of Encoded Source Electromagnetic Sounding
}

\author{
Weibing Luo, Haitao Han \\ Gansu Nonferrous Geological \\ Survey Institute \\ LanZhou, China \\ lwbcsu@163.com
}

\author{
Mei Li \\ School of Information Engineering, \\ University of Geosciences (Beijing) \\ BeiJing, China \\ maggielimei@163.com
}

\author{
Jingtian Tang \\ School of Geosciences and Info- \\ Physics, Central South University \\ ChangSha, China \\ jttang@mail.csu.edu.cn
}

\begin{abstract}
The encoded source electromagnetic sounding method based on circular correlation identification and the calculation method of whole zone apparent resistivity are put forward. Using the inverse repeated $M$ sequence as the excitation signal, the encoded current from the source is through the grounded electrodes of horizontal electric dipole, the electric field with the same horizontal direction of the transmitter dipole or the vertical magnetic field is measured in the equatorial array. The excitation current and the received electric voltage signal or the vertical magnetic field are simultaneously recorded in the time series at the sampling rate using the same acquisition equipment, and using circular cross-correlation method to obtain the earth frequency response, then the whole zone apparent resistivity spectrum are calculated according to the frequency response and measurement parameters of the electromagnetic sounding array. The results show that the encoded source electromagnetic sounding based on circular cross-correlation method to obtain the whole zone apparent resistivity spectrum, raises the efficiency of electromagnetic sounding and improve the whole zone apparent resistivity frequency resolution, and due to the adoption of the circular cross-correlation method and the current signal of inverse repeated $M$ sequence is an even multiple of power frequency, can effectively suppress the power frequency interference, so this method has enhanced anti-jamming ability.
\end{abstract}

Keywords-encoded source electromagnetic sounding; circular cross correlation; Whole zone apparent resistivity; frequency response; inverse repeated $M$-sequence

\section{INTRODUCTION}

Controlled source frequency domain electromagnetic sounding method has become an effective method to obtain the geoelectric section. This method has been widely used in metal minerals exploration, oil and gas, coal, geothermal, engineering geological survey and other fields. At present commonly used frequency domain electromagnetic sounding method, by changing the frequency, one frequency and then another, sweep all preset frequency table to measure the resistivity of the exploration region, using the observed two orthogonal horizontal component of Ex and Hy to estimate the Cagniard resistivity.

In order to improve the exploration efficiency, academician He Ji-shan of Central South University proposed Wide Field Electromagnetic Method(WFEM) using $2^{\text {n }}$ sequence pseudo-random multi frequency wave as the excitation waveform, it is a wide band signal, so in one excitation, several main frequency apparent resistivity can be obtained, improved the work efficiency. Wide Field Electromagnetic Method(WFEM) using electric field horizontal component of Ex to calculate the whole zone apparent resistivity, which represents the development of the controlled source electromagnetic sounding.

Duncan $^{[1]}$ et al. (1980) tried the broadband electromagnetic sounding method based on pseudo random binary sequence (PRBS) as the signal source, the encoded current injected ground by horizontal electric dipole, the vertical magnetic field $\mathrm{Hz}$ was measured in the far field, and using crosscorrelation method to obtain the impulse response, are transformed into the frequency domain, the broadband amplitude and phase frequency response then obtained. In the experiment, the electric dipole length was $20.5 \mathrm{~km}$, the maximum transmitting current was $5 \mathrm{~A}$, and the maximum transmission-receiver distance was $85 \mathrm{~km}$, and the geoelectric section depth of the $20 \mathrm{~km}$ were obtained. Anton Ziolkowski et al. (2007) developed a multitransient electromagnetic method (MTEM).based on the PRBS encoded source. The electric field components in the same direction with the transmit dipole were measured, simultaneous recording of the excitation current and the voltage of electric field in time series, deconvolution the two time series to obtain impulse response of the detected geoelectric system and using the impulse response peak time to calculate apparent resistivity and other electromagnetic parameter for interpretation. The commercial applications for onshore and offshore oil and gas exploration was successful.

On this basis, this paper presents a new method to directly obtain the frequency response of the geoelectric system to be detected. Based on the circular cross-correlation method, the system frequency response is recovered by the input and output time series, and has a high frequency resolution. It is an innovation of the traditional frequency domain electromagnetic sounding method, which can improve the working efficiency, formation resolution and anti-interference ability.

\section{THEORY AND METHOD}

The encoded source frequency domain electromagnetic sounding method proposed in this paper, using inverse 
repeated $\mathrm{M}$ sequence as signal waveform, the encoded current from the source is through the grounded electrodes of horizontal electric dipole, the receiver end electric field voltage or the vertical magnetic field and the transmit end current signal are synchronous sampled using the same record equipment, and using circular cross-correlation method to obtain the earth frequency response(Fig.1), then the whole zone apparent resistivity spectrum are calculated according to the earth frequency response and measurement parameters of the electromagnetic sounding array.

Inverse repeated $M$ sequences are generated by shift registers. After setting the $\mathrm{N}$ bit register and the fixed feedback bits, the generated inverse repeated $M$ sequence is fixed. The sequence with different register initial states have different time delay, but have the same spectrum distribution(Fig. 2).The correlation peak is inverse repeated(Fig. 3).

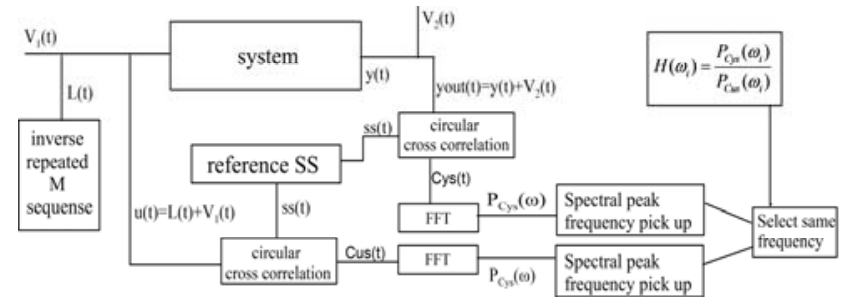

Fig.1 workflow of system identification based on inverse repeated M-

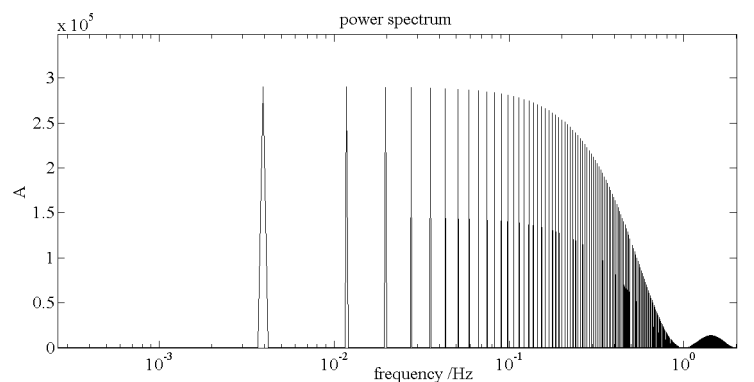

Fig.2 workflow of system identification based on inverse repeated Msequence

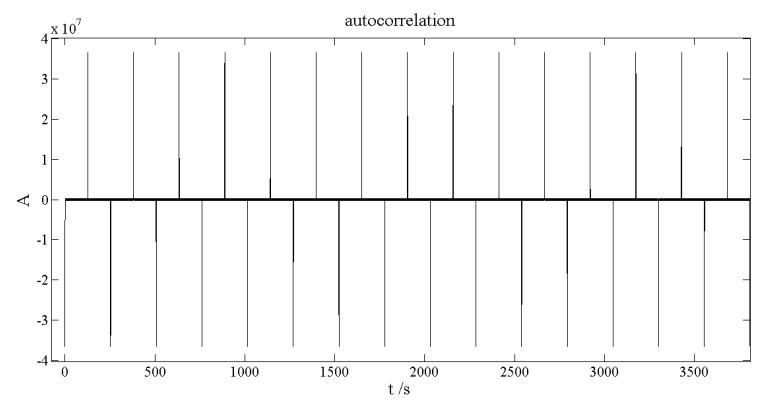

Fig. 3: auto correlation of inverse repeated M-sequence

For cross-correlation calculation of two time series, using non-circular cross-correlation calculation, need to fill in the tail of the sequence with zero, to participate equal length sequences for calculation, the calculation results will produce unwanted interference on both sides of pulse peaks, the multiperiodic sequence can suppress interference on both sides of the pulse peak, but the interference will still exist. And the correlation peak amplitude will decreases with the time delay increases(Fig.4).

The interference of both sides of the correlation peak can be effectively suppressed by the circular cross-correlation method. In the circular cross-correlation method calculation, each time series linked up end to end, so in the calculation the time sequence cyclic shift, without zero padding. The interference of both sides of the correlation peak is much more stable, and the amplitude of the correlation peak is

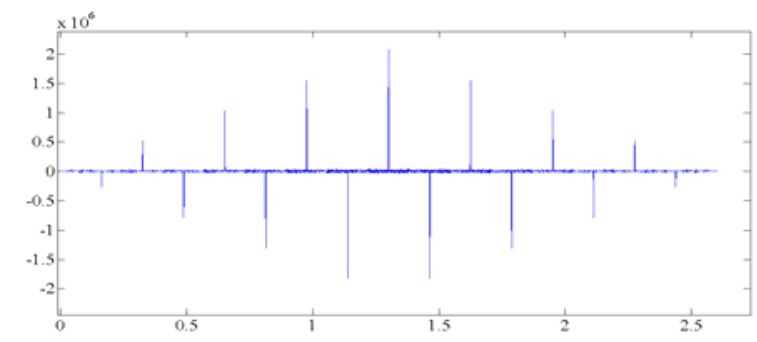

Fig.4 non-circular auto correlation of inverse repeated M-sequence

equal(Fig.5). This characteristic has the advantage in the power spectrum superposition of multi-periodic signal.

By time series of electric field component Ex or vertical magnetic field component $\mathrm{Hz}$ and transmit current time series, using circular cross-correlation method to identify the frequency response of the geoelectric system, introduction of a reference signal can suppress the interference, the calculation as follows:

The first step, to calculate the circular cross correlation of input current time sequence with the reference signal and the output electromagnetic response time sequence with the reference signal, the reference signal can be directly extracted

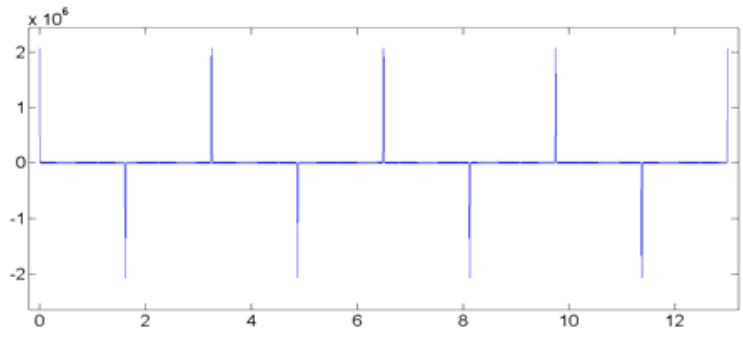

Fig.5 circular auto correlation of inverse repeated M-sequence

from the current time series or generated by software:

$$
\begin{aligned}
& C_{\text {us }}(t)=\operatorname{cxcorr}(\mathrm{u}(t), S S(t)) \\
& C_{y s}(t)=\operatorname{cxcorr}(\operatorname{yout}(t), S S(t))
\end{aligned}
$$

In the second step, the fast Fourier transform of the two circular cross-correlation time series is calculated, to obtain the power spectrum: 


$$
\begin{aligned}
& P_{\text {Cys }}(\omega)=\operatorname{FFT}\left(C_{y s}(\mathrm{t})\right) \\
& P_{\text {Cus }}(\omega)=\operatorname{FFT}\left(C_{u s}(\mathrm{t})\right)
\end{aligned}
$$

The third step, select the same frequency output power spectrum amplitude divided by input power spectrum amplitude, get the frequency response of amplitude and phase:

$$
\begin{aligned}
\mathrm{H}(\omega) & =\frac{P_{\text {Cys }}(\omega)}{P_{\text {Cus }}(\omega)} \\
\varphi(\omega) & =\bmod \left(\left(\varphi_{\text {Pcys }}(\omega)-\varphi_{\text {Pcus }}(\omega)\right), \pi\right)-\pi
\end{aligned}
$$

In the above formula, cxcorr is the circular crosscorrelation operation, FFT is fast Fourier transform, mod is a modular operator, $u(t)$ is the system input signal time series (including noise $\left.V_{1}(\mathrm{t})\right)$, yout $(t)$ is time series of the system output electric field signal Ex or vertical magnetic field signal $\mathrm{Hz}$ (including noise $V_{2}(\mathrm{t})$ ), SS is the reference signal without the interference extracted from transmit current waveform, $P_{C y s}(\omega)$ is the cross power spectrum of reference signal SS and the output signal yout $(t), P_{\text {Cus }}(\omega)$ is the cross power spectrum of reference signal $S S$ and the input signal $u(\mathrm{t}), \varphi(\omega)$ is the phase.

When the observed field value is the time series of the electric field component Ex, the observed value is the voltage time series between the receiver electrodes, and the $\mathrm{MN}$ is the electrode spacing of the receiver electric field, so the calculation process is changed from the above calculation process (5):

$$
\mathrm{Z}_{\mathrm{Ex}}(\omega)=\frac{P_{C_{V_{e x}} \mathrm{~s}}(\omega)}{P_{C_{I_{A B}} \mathrm{~s}}(\omega)}
$$

In above formulation, $P_{C_{V_{\mathrm{S}}} s}(\omega)$ is the cross power spectrum of the reference signal $S S$ and the receiving signal voltage $V_{E x}$, $P_{C_{A B} S}(\omega)$ is the cross power spectrum of the reference signal SS and the current signal $I_{A B}(\mathrm{t})$ of horizontal electric dipole source AB.

We know that the electric field generated by horizontal electric dipole source on surface of uniform half space is:

$$
E_{x}=\frac{\rho I d L}{2 \pi r^{3}}\left[\left(1-3 \sin ^{2}(\varphi)\right)+\left(1-i k_{1} r\right) \exp \left(i k_{1} r\right)\right]
$$

In the formula, $\rho$ is the resistivity of the uniform earth , $I$ is the transmitting current, $r$ is the distance from the midpoint of the receiving electrode $\mathrm{MN}$ to the midpoint of the transmit dipole $\mathrm{AB}$, and $\varphi$ is the angle between $\mathrm{R}-\mathrm{T}$ line and the line of $\mathrm{AB}$ bipole, $d L$ is $\mathrm{AB}$ dipole spacing. $k_{1}^{2}=i \omega \mu_{0} / \rho$ is wave number of uniform earth.

Formula (8) divided by the current $I$ and equivalent to the formula (7) correspondingly, available:

$$
\begin{gathered}
\mathrm{Z}_{E x}(\omega)=\frac{\rho(\omega) d L \cdot M N}{2 \pi r^{3}} C_{E x} \\
C_{E x}=\left(1-3 \sin ^{2}(\varphi)\right)+\left(1-i k_{1} r\right) \exp \left(i k_{1} r\right)
\end{gathered}
$$

$\mathrm{Z}_{E x}(\omega)$ is the impedance of the earth obtained by circular cross-correlation of observing voltage value of the electric field Ex and the injected current $I$. Thus the whole zone apparent resistivity spectrum can be got by formula (11):

$$
\rho_{E x}(\omega)=\frac{2 \pi r^{3} \cdot Z_{E x}(\omega)}{d L \cdot M N \cdot C_{E x}}
$$

In formula, $C_{E x}$ is also a function of the earth resistivity, therefore, the formula (11) of the whole zone apparent resistivity needs to be solved by iterative method. This algorithm has been verified in the field data processing.

When the observed electromagnetic field value is the vertical magnetic field component $\mathrm{Hz}$, the calculation procedure from formula (1) to formula (4) is same as above, and formula (5) is changed into formula (12):

$$
\mathrm{Z}_{H z}(\omega)=\frac{P_{C H z s}(\omega)}{P_{C I_{A B}}(\omega)}
$$

In formula, $P_{\text {CHzs }}(\omega)$ is the cross power spectrum of the reference signal $S S$ and the receiving vertical magnetic field signal $\mathrm{Hz} ; P_{C_{A B} \mathrm{~S}}(\omega)$ is the cross power spectrum of the reference signal $S S$ and current signal $I_{A B}(\mathrm{t})$ through the horizontal electric dipole source AB.

We know that the vertical magnetic field $\mathrm{Hz}$ produced by horizontal electric dipole source on homogeneous earth surface is:

$$
H_{\mathrm{Z}}=-\frac{3 I d L}{2 \pi k_{1}^{2} r^{4}} \sin \varphi\left[1-\left(1-i k_{1} r-\frac{1}{3}\left(k_{1} r\right)^{2}\right) \exp \left(i k_{1} r\right)\right]
$$

In the formula, $I$ is the transmit current, $r$ is the distance of magnetic field receiving midpoint $\mathrm{P}$ to the midpoint of the dipole $\mathrm{AB}, \varphi$ is the angle between $\mathrm{R}-\mathrm{T}$ line and the extension line of dipole AB. $d L$ is length of dipole AB. $k_{1}^{2}=i \omega \mu_{0} / \rho$ is the wave number of the homogeneous earth, $\mu_{0}$ is the permeability.

Formula (13) divided by the current $I$ and equivalent to formula (12) correspondingly, available:

$$
\begin{aligned}
& \mathrm{Z}_{H_{Z}}(\omega)=-\frac{3 d L \cdot \rho(\omega)}{i \omega \mu_{0} 2 \pi r^{4}} \sin \varphi C_{H_{Z}} \\
& C_{H z}=1-\left(1-i k_{1} r-\frac{1}{3}\left(k_{1} r\right)^{2}\right) \exp \left(i k_{1} r\right)
\end{aligned}
$$

$\mathrm{Z}_{\mathrm{Hz}}(\omega)$ is the frequency response of the earth obtained by circular cross-correlation of the observing vertical magnetic field $\mathrm{Hz}$ and the excitation current $I$. Thus the whole zone apparent resistivity spectrum can be got by formula (16):

$$
\rho_{H_{Z}}(\omega)=\frac{2 \pi \omega \mu_{0} r^{4} \cdot Z_{H_{Z}}(\omega)}{3 i \cdot d L \cdot \sin \varphi \cdot C_{H_{Z}}}
$$

In formula, $C_{\mathrm{Hz}}$ is also a function of the earth resistivity, so the formula (16) needs to be solved by iteration method.

Due to the excitation signal of inverse repeated $M$ sequence is a broadband signal, so the above method can get a series of frequency of apparent resistivity spectrum. In order to obtain high quality data, it is benefit to transmit and receive multiple cycles of inverse repeated $M$ sequence of encoded 
source signal. Longer time records are helpful to improve the signal to noise ratio.

\section{EXAMPLES}

Using the method proposed in this paper, we deal with a sounding station of wide field electromagnetic measurement data. Excitation current waveform is 2 n series pseudo random sequence, which have 7 main frequencies. Measurement parameters are: the length of grounded electrodes of horizontal electric dipole $A B=1004 \mathrm{~m}$, receiver dipole $M N=100 m$, transmit and receive distance $r=16593.6 \mathrm{~m}$. Azimuth of $\mathrm{R}-\mathrm{T}$ line and the extension line of dipole $\mathrm{AB}$ is 79.84 degrees. The time series of the received electric field and the excitation current were recorded simultaneously. In order to get as far as possible the frequency distribution, 4 excitation were conducted, the fundamental frequency is $0.75,1,96$ and $128 \mathrm{~Hz}$ separately, the sampling rate is 4800 sps for 0.75 and $1 \mathrm{~Hz}, 38400$ sps for 96 and $128 \mathrm{~Hz}$. Maximum transmit current 25.4A.Using circular cross-correlation method, the frequency response were identified for each group data. Then according to the formula (11) the whole zone apparent resistivity were calculated. The results as shown in the fig. 6 , there are 315 frequencies of magnitude and phase were obtained, the frequency resolution is much higher than that of conventional processing method.

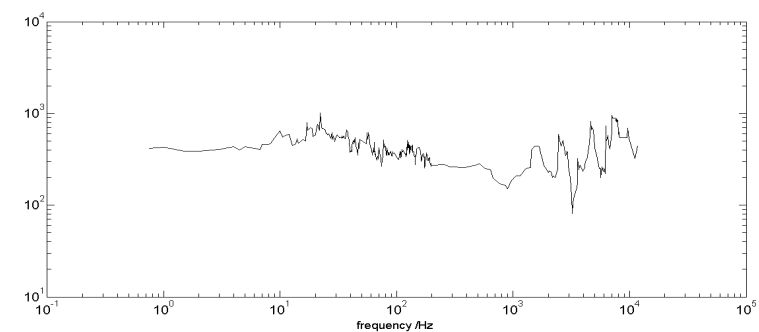

Fig.6 whole zone apparent resistivity of one WFEM sounding

\section{CONCLUSIONS}

Using encoded source current and multi cycle continuous excitation, by the method proposed in this paper, we can get broadband earth whole zone apparent resistivity spectrum by recording time series of electric field observation of Ex or vertical magnetic field $\mathrm{Hz}$ in certain transmit-receiver distance, to achieve the purpose of electromagnetic sounding. The apparent resistivity spectrum has higher frequency resolution, and due to the adoption of the circular cross correlation identification technology, this method has certain suppression for interferences and has strong anti-interference ability.

\section{ACKNOWLEDGMENTS}

The authors wish to thank the support of National Natural Science Foundation of China(No.41374185), thanks also to Dr. LI di-quan and Jiang qi-yun of Central South University for share the WFEM data.

\section{REFERENCE}

[1] Zhdanov, Michael S., "Electromagnetic geophysics: Notes from the past and the road ahead," Geophysics, 75(5), pp. 75A49-75A66, 2010. 